\title{
5. Soziale Isolation und Einsamkeit bei Frauen und Männern im Verlauf der zweiten Lebenshälfte
}

\author{
Oliver Huxhold und Heribert Engstler
}

\section{Kernaussagen}

Die Risiken sozialer Isolation und Einsamkeit sind unterschiedlich hoch und entwickeln sich mit dem Älterwerden im Verlauf der zweiten Lebenshälfte verschieden: Während das Isolationsrisiko zwischen dem 40. und 90. Lebensjahr relativ kontinuierlich von vier auf 22 Prozent steigt, verläuft das Einsamkeitsrisiko u-förmig. Vom Alter 40 bis Mitte 60 sinkt es, danach nimmt es wieder zu, ist aber selbst im sehr hohen Alter, mit 90 Jahren, mit rund elf Prozent nicht höher als mit 40 Jahren.

Das Isolationsrisiko unterscheidet sich im Alternsverlauf zwischen Frauen und Männern: Bei Männern steigt das Risiko sozialer Isolation über die betrachtete Altersspanne zwischen 40 und 90 Jahren relativ gleichmäßig von fünf auf 20 Prozent an. Frauen erleben zunächst einen schwächeren Risikoanstieg, der sich im Rentenalter jedoch beschleunigt, so dass sie im Alter ab Ende 70 ähnlich häufig sozial isoliert sind wie Männer. Zuvor haben Frauen mehr als drei Lebensjahrzehnte lang vom Alter Anfang 40 bis Mitte 70 ein geringeres Isolationsrisiko als Männer.

Auch das Einsamkeitsrisiko unterscheidet sich im Alternsverlauf zwischen Frauen und Männern: Im mittleren Erwachsenenalter zwischen 40 und 60 Jahren sind Männer etwas häufiger einsam als Frauen. Der Geschlechterunterschied nimmt mit steigendem Alter jedoch ab und dreht sich im Verlauf des Rentenalters um, so dass im hohen Alter mehr Frauen als Männer einsam sind. Mit 90 Jahren haben Frauen ein Risiko von 14 Prozent einsam zu sein. Bei Männern in diesem Alter beträgt das Einsamkeitsrisiko neun Prozent.

Bei jüngeren Geburtskohorten steigt das Isolationsrisiko mit dem Älterwerden nicht mehr so hoch an wie bei den älteren Geburtsjahrgängen. Das Einsamkeitsrisiko ist in den später geborenen Jahrgängen weniger stark mit dem Älterwerden verknüpft. Der soziale Wandel hat die Unterschiede zwischen Frauen und Männern nicht verändert: Während bei den 1930 bis 1939 geborenen Menschen das Isolationsrisiko bis in das Alter von Mitte 70 stieg, endet der Altersanstieg des Isolationsrisikos bei den 1950 bis 1959 Geborenen vorläufig bereits im Alter Mitte 60 auf einem niedrigeren Niveau. Beim Einsamkeitsrisiko zeigt sich für die 1950 bis 1959 Geborenen ein niedrigeres Ausgangsniveau im mittleren Erwachsenenalter und eine Abflachung des u-förmigen Alternsverlaufs. Voraussichtlich werden die dann 70- bis 80-Jährigen nicht häufiger einsam oder sozial isoliert sein als die heutigen 70- bis 80-Jährigen. Zudem ist bislang keine Angleichung in den Alternsverläufen zwischen Frauen und Männern erkennbar. 


\subsection{Einleitung}

Mit anderen Menschen verbunden zu sein, das eigene Leben mit anderen $\mathrm{zu}$ teilen, gute und enge Beziehungen zu haben, ist allen Frauen und Männern ein angeborenes Bedürfnis (Baumeister \& Leary 1995). Aus diesem Grund sind soziale Isolation und Einsamkeit für viele Menschen zutiefst stressvolle Erfahrungen, die mit erheblichen Gesundheitsrisiken verbunden sind (Hawkley \& Cacioppo 2010). Insbesondere lange Phasen der Einsamkeit erhöhen unter anderem das Risiko von Schlafstörungen, Depressionen und kardiovaskulären Erkrankungen und gehen letztlich sogar mit einem erhöhten Mortalitätsrisiko einher (Luo, Hawkley, Waite, \& Cacioppo 2012).

In der öffentlichen Debatte werden die Begriffe „Soziale Isolation“ und „Einsamkeit“ häufig synonym verwendet. Die Forschung hat jedoch wiederholt gezeigt, dass es sich um unterschiedliche Phänomene handelt (Hawkley \& Cacioppo 2010). Je nach Persönlichkeit unterscheiden sich Menschen darin, wie viel soziale Einbettung sie bevorzugen. So gibt es zum Beispiel Menschen, die sich auf eine enge Freundschaft konzentrieren, und solche, die einen großen Kreis aus mehr oder weniger engen Freunden und Bekannten pflegen (Miche, Huxhold, \& Stevens 2013). Im Gegensatz $\mathrm{zu}$ solchen gewünschten Unterschieden in der $\mathrm{Zu}$ sammensetzung und Beziehungsintensität sozialer Netzwerke zwischen Personen, bezeichnet soziale Isolation einen bedeutenden Mangel im Kontakt $\mathrm{zu}$ anderen Menschen. Sie kann von außen beobachtet werden, unabhängig von der subjektiven Bedeutung für das Individuum. Menschen, die viel Zeit allein verbringen und wenig mit anderen Personen interagieren, können als sozial isoliert bezeichnet werden (Hawthorne 2006). Einsamkeit ist hingegen eine sehr subjektive Erfahrung. Sie beschreibt das zutiefst unangenehme Gefühl, dass die sozialen Beziehungen, die man pflegt, und der persönliche Austausch mit anderen Menschen nicht den eigenen Bedürfnissen nach Zugehörigkeit und Geborgenheit entsprechen (Perlman \& Peplau 1981). Demzufolge haben Menschen, die sozial isoliert sind, zwar eine höhere Wahrscheinlichkeit sich einsam zu fühlen, es gibt aber auch viele Personen, die von sich aus mit nur sehr wenigen Menschen einen engen Austausch pflegen und mit dem Alleinsein gut zurechtkommen. Andererseits können Menschen sich einsam fühlen, die objektiv gesehen über viele soziale Kontakte verfügen, weil ihnen in diesen Beziehungen emotionale Tiefe und Verständnis fehlen.

In den letzten Jahrzehnten hat sich fast in allen westlichen Industrienationen eine starke öffentliche Debatte um die Themen soziale Isolation und Einsamkeit herausgebildet. Zwei Befürchtungen stehen innerhalb dieser Diskussion im Zentrum. Zum einen befürchten viele Menschen, dass im Alter das Risiko, sozial isoliert und/oder einsam zu sein, enorm hoch sei (Drinkuth 2018) - fast so als sei Einsamkeit eine garantierte Begleiterscheinung des hohen Erwachsenenalters. Zum anderen wird häufig die Meinung vertreten, dass der Anteil der Menschen, die unter sozialer Isolation und Einsamkeit leiden, in den letzten Jahren stark zugenommen habe (Drinkuth 2018; Will 2017). In einigen Veröffentlichungen wird sogar von einer "Epidemie der Einsamkeit“ gesprochen, die immer weitere Teile der Bevölkerung insbesondere unter den älteren Menschen erfassen wird (Drinkuth 2018; Will 2017). Doch entsprechen diese beiden häufig geäußerten Befürchtungen der Wirklichkeit? Nehmen die Risiken, sozial isoliert zu sein und einsam zu sein, wirklich mit steigendem $\mathrm{Al}$ ter zu?

Um die letztgenannte Frage beantworten zu können, bedarf es längsschnittlicher Analysen. Das Isolationsrisiko oder das Einsamkeitsrisiko für die gleichen Personen muss über einen längeren Zeitraum hinweg mehrfach gemessen werden, um beurteilen zu können, ob diese Risiken tatsächlich im Zuge des Älterwerdens ansteigen (siehe Kapitel 2 Design, Inhalte und Methodik des Deutschen Alterssurveys in diesem Band). Leider sind bisherige Forschungsarbeiten zum Zusammenhang zwischen Alter und Einsamkeit jedoch 
mit wenigen Ausnahmen (z. B. Böger \& Huxhold 2018) querschnittlicher Natur. Querschnittliche Analysen vergleichen das Ausmaß von Einsamkeit zwischen verschiedenen Altersgruppen zu einem einzigen Zeitpunkt. Aufgrund solcher Untersuchungen lässt sich nicht unterscheiden, ob eventuelle Altersgruppenunterschiede tatsächlich auf Effekte des Älterwerdens zurückzuführen sind oder ob sich diese Ungleichheiten auf Grund von unterschiedlichen Lebensbedingungen verschiedener Geburtskohorten ergeben. Zudem muss man bedenken, dass die Älteren keine homogene Gruppe sind. Lebensbedingungen, die zur Ausbildung eines erhöhten Risikos für soziale Isolation und für Einsamkeit führen können - wie zum Beispiel das Fehlen einer festen Partnerschaft -, unterscheiden sich beispielsweise zwischen Frauen und Männern und das in unterschiedlichem Maße in unterschiedlichen Lebensphasen.

\section{Unterschiede zwischen Frauen und Männern}

Generell ergab die bisherige Forschung relativ uneinheitliche Ergebnisse in Bezug auf Unterschiede im Ausmaß sozialer Integration zwischen Frauen und Männern. Während einige Studien zeigten, dass Frauen größere soziale Netzwerke pflegen als Männer (Suanet \& Huxhold 2018), fanden andere Untersuchungen wiederum keinerlei Unterschiede zwischen den Geschlechtern (Antonucci, Lansford, Akiyama, Smith, Baltes, Takahashi, Fuhrer, \& Dartigues 2002). Auch bezüglich der Geschlechterunterschiede in der Verbreitung von Einsamkeit sind die Studienergebnisse nicht eindeutig und zudem abhängig von dem jeweils verwendeten Messinstrument (von Soest, Luhmann, Hansen, \& Gerstorf 2018). Dieses insgesamt uneinheitliche Bild lässt sich vermutlich dadurch erklären, dass die sozialen Beziehungen von Frauen und Männern gleichzeitig von verschiedenen Einflüssen gestaltet werden, die sich unterschiedlich auf das Isolationsund Einsamkeitsrisiko auswirken und in der Summe auch gegenseitig aufheben können. So sind auf der einen Seite im höheren Lebensalter Risi- kofaktoren für soziale Isolation und Einsamkeit wie beispielsweise einschränkende Krankheiten, Verwitwung und finanzielle Nöte bei Frauen stärker ausgeprägt als bei Männern (Antonucci et al. 2002). Aus einer Ressourcenperspektive heraus würde man daher mutmaßen, dass sich Geschlechterunterschiede mit dem Alter vergrößern, da diese gesundheits- und verlustbezogenen Risiken für Einsamkeit und soziale Isolation für Frauen stärker zunehmen als für Männer. Auf der anderen Seite jedoch investieren Frauen stärker als Männer in soziale Beziehungen und pflegen häufigeren Kontakt zu ihren Familien und insbesondere $\mathrm{zu}$ ihren Freundinnen und Freunden (Sander, Schupp, \& Richter 2017). Frauen scheinen möglicherweise aus diesem Grund besser in der Lage zu sein, auch mit fortschreitendem Alter eher auf soziale Unterstützung aus ihrem sozialen Netzwerk zurückgreifen zu können als Männer (Fischer \& Beresford 2015). Betrachtet man also die Entwicklung der Risiken für soziale Isolation und Einsamkeit aus einer Perspektive, die diese kumulative Investition in soziale Netzwerke über die Lebensspanne berücksichtigt, so sind ganz im Gegensatz zu einer Ressourcenperspektive Männer mit fortschreitendem Alter eher gefährdet als Frauen. Diese Vermutung könnte insbesondere auf später geborene Geburtskohorten zutreffen, wie wir im nächsten Abschnitt darlegen werden.

\section{Wandel geschlechtsspezifischer Verläufe der Risiken von Einsamkeit und sozialer Isolation}

In der öffentlichen Debatte wird mitunter vor einer herannahenden „Epidemie der Einsamkeit“ gewarnt, also einer starken Zunahme der sozialen Isolation und Einsamkeit bei den später geborenen Geburtsjahrgängen (Drinkuth 2018). Begründet wird dies häufig mit dem beobachteten Anstieg von Kinderlosigkeit, angewachsenen Scheidungsraten und vergrößerten Wohnentfernungen zwischen Familienmitgliedern in den letzten Jahrzehnten (Mahne \& Huxhold 2017; Engstler \& Tesch-Römer 2010). Dabei wird in dieser Debatte häufig über- 
sehen, dass trotz der wachsenden Wohnentfernungen wahrscheinlich auch auf Grund der Nutzung moderner Kommunikationsmittel die Qualität der Familienbeziehungen sehr gut geblieben ist (Mahne \& Huxhold 2017). Zudem zeigt sich, dass die sozialen Netzwerke heutzutage viel stärker als früher Freundinnen und Freunde beinhalten und diese immer mehr soziale Unterstützungsleistungen übernehmen, die vormals Familienmitgliedern vorbehalten waren (Böger, Wetzel, \& Huxhold 2017; Huxhold, Mahne, \& Naumann 2010; Suanet \& Antonucci 2017). Eine Studie, die Geschlechterunterschiede über verschiedene Kohorten hinweg hinsichtlich der längsschnittlichen Entwicklung (über 17 Jahre) von Freundschaftsbeziehungen untersuchte, ist die Longitudinal Aging Study Amsterdam (LASA; Stevens \& van Tilburg 2011). Auch diese Studie bestätigte eine wachsende Bedeutung von Freundschaften und deren Aufrechterhaltung im Alter innerhalb jüngerer Kohorten und traf zudem Aussagen über typische Entwicklungsverläufe von Freundschaften für Frauen und Männer. So zeigten die Autorin und der Autor nicht nur, dass Frauen insgesamt mehr Freundschaften berichten als Männer und diese länger aufrechterhalten, sondern auch, dass dieser Geschlechterunterschied über die Kohorten hinweg zunahm. Diese Freundschaftsbeziehungen könnten Frauen nutzen, um eventuelle Lücken in Familienbeziehungen auszugleichen. Passend dazu fanden Perrig-Chiello und Kollegen (2015), dass nur bei verwitweten Frauen, aber nicht bei verwitweten Männern, ein Mangel an sozialen Kontakten im Jahr 2011 signifikant seltener berichtet wurde als bei einer befragten Kohorte im Jahr 1979 (Perrig-Chiello, Spahni, Hopflinger, \& Carr 2016). Die oben zitierten Befunde sprechen für die Vermutung, dass es Frauen insgesamt etwas besser als Männern gelungen ist, sich den veränderten Familienbeziehungen anzupassen. Über Geburtskohorten hinweg lässt sich aus dieser Perspektive erwarten, dass sich die Alternsverläufe der Risiken sozialer Isolation und Einsamkeit in später geborenen Geburtskohorten positiver für Frauen als für Männer entwickeln.

\section{Fragestellungen}

Im Zentrum dieses Kapitels stehen die Fragen, wie sich mit dem Älterwerden im Verlauf der zweiten Lebenshälfte die Risiken sozial isoliert und/oder einsam zu sein, entwickeln und ob diese Risiken für Frauen und Männer unterschiedlich sind. Darüber hinaus wird geprüft, ob es in Deutschland einen gesellschaftlichen Wandel zu mehr Isolation und Einsamkeit im Alter gibt und ob sich dieser bei Frauen und Männern unterscheidet. Der Beitrag beschreitet in mehrerer Hinsicht Neuland. Zunächst werden erstmalig längsschnittliche Entwicklungsverläufe hinsichtlich der Risiken für soziale Isolation und Einsamkeit getrennt für Frauen und Männer betrachtet und nicht nur Altersgruppen miteinander verglichen. Hierbei gehen wir davon aus, dass Frauen im mittleren Lebensalter möglicherweise im Vorteil sind, da sie tendenziell stärker in ihre sozialen Netze investieren. Mit steigendem Lebensalter und einer möglichen $\mathrm{Zu}$ nahme von funktionalen Gesundheitseinschränkungen sowie einem erhöhten Verwitwungsrisiko könnte sich dieser Vorteil jedoch abschwächen, da Frauen häufiger als Männer im höheren Alter von diesen negativen Entwicklungen und Lebensereignissen betroffen sind. Zudem könnten Frauen den historischen Wandel in den Familien- und Paarbeziehungen möglicherweise besser bewältigen als Männer, da sie in einem stärkeren Maße Freundschaften pflegen. Geschlechterunterschiede in den längsschnittlichen Alternsverläufen von sozialer Isolation und Einsamkeit könnten sich dementsprechend zu Gunsten von Frauen verlagern. 


\subsection{Methoden}

\section{Stichprobe}

Die empirische Untersuchung der geschlechtsspezifischen Alternsverläufe des Einsamkeitsrisikos und des Risikos sozialer Isolation stützt sich auf die Paneldaten des Deutschen Alterssurveys (DEAS) der Jahre 1996 bis 2017. In den Jahren 1996, 2002, 2008 und 2014 wurden jeweils unabhängige, für Deutschland repräsentative Einwohnermeldeamtsstichproben der in Privathaushalten lebenden 40- bis 85-jährigen Bevölkerung gezogen, die nach ihrer ersten Befragung in den Längsschnitt aufgenommen und in den Folgewellen erneut interviewt wurden. Am Deutschen Alterssurvey haben bislang mehr als 20000 Studienteilnehmende mit bis zu sechs Messzeitpunkten teilgenommen. Nähere Informationen zum Stichprobendesign enthält Kapitel 2 Design, Inhalte und Methodik des Deutschen Alterssurveys. Einschränkend muss darauf hingewiesen werden, dass im Deutschen Alterssurvey nahezu keine Heimbewohnerinnen und -bewohner enthalten sind, da Untersuchungsteilnehmer nach einem Umzug ins Heim überwiegend nicht mehr befragt werden können. Darüber hinaus erwies sich bei insgesamt geringer Selektivität der Panelmortalität insbesondere das Bildungsniveau als ein relevanter Einflussfaktor auf die Bleibewahrscheinlichkeit in der Studie (vgl. Kapitel 2 Design, Inhalte und Methodik des Deutschen Alterssurveys in diesem Band). Da wir für Heimbewohnerinnen und -bewohner und Niedriggebildete ein erhöhtes Risiko sozialer Isolation und Einsamkeit erwarten, sind diese beiden Selektivitäten der Panelmortalität bei der Interpretation der Ergebnisse zu berücksichtigen. In die vorliegende Untersuchung sind alle Studienteilnehmerinnen und -teilnehmer einbezogen, für die mindestens

1 Grundlage der international gebräuchlichen Einsamkeitsskala sind drei negativ und drei positiv formulierte Aussagen, zu denen jeweils anzugeben ist, ob diese auf einen selbst „genau“, „eher", „eher nicht" oder "gar nicht“ zutreffen (4-stufige Antwortskala). Die Items lauten: „Ich vermisse Leute, bei denen ich mich eine Beobachtung des Isolations- beziehungsweise Einsamkeitsrisikos vorliegt und die prinzipiell wiederholt befragt werden konnten. Um den Einfluss selektiver Panelmortalität zu verringern, wurden auch jene Studienteilnehmerinnen und -teilnehmer einbezogen, die nur an einer Befragungswelle des Deutschen Alterssurveys teilgenommen haben. Die Untersuchung des Risikos sozialer Isolation im Alternsverlauf stützt sich damit auf 39186 Messungen bei 19969 Personen. Für die Untersuchung des Einsamkeitsrisikos liegen mit 31532 Messungen bei 16151 Personen etwas geringere Fallzahlen vor, da die Einsamkeitsindikatoren im zusätzlichen Selbstausfüller-Fragenbogen erhoben wurden, den nicht alle Interviewten ausgefüllt haben.

\section{Indikatoren}

In unserer Untersuchung messen wir soziale Isolation anhand der Größe des persönlichen Netzwerks, bestehend aus den Personen, die den Individuen nach eigener Aussage persönlich wichtig sind und mit denen sie regelmäßig Kontakt haben. Nur wenn sie niemanden oder maximal eine Person nennen können, auf die das zutrifft, werten wir dies als soziale Isolation. Dabei zählen wir Haushaltsmitglieder des Individuums grundsätzlich zu seinem Netzwerk. Soziale Isolation wird für die Analysen also als dichotome Variable mit den beiden Ausprägungen 1 (isoliert) und 0 (nicht isoliert) verwendet. Zur Bestimmung der Einsamkeit stützen wir uns auf die im Deutschen Alterssurvey verwendete Einsamkeitsskala in der Kurzform von de Jong Gierveld und van Tilburg (2006) mit einem Mittelwert aus sechs Items. ${ }^{1}$ Die Skalenausprägungen können vom Wert 1 (gar nicht einsam)

wohl fühle“, „Ich fühle mich häufig im Stich gelassen“, „Ich vermisse Geborgenheit und Wärme“, „Es gibt genug Menschen, die mir helfen würden, wenn ich Probleme habe“, „Ich kenne viele Menschen, auf die ich mich wirklich verlassen kann“, „Es gibt genügend Menschen, mit denen ich mich eng verbunden füh- 
bis 4 (sehr einsam) reichen. Zu den Einsamen zählen wir Personen mit einem Skalenwert, der größer als 2,5 ist, sich also in der oberen Hälfte des möglichen Wertebereichs der Skala befindet. Mit dieser Aufteilung der Skalenwerte erfassen wir Einsamkeit als dichotome Variable mit den beiden Ausprägungen 1 (einsam) und 0 (nicht einsam). Zur Interpretation der folgenden Ergebnisse muss deshalb beachtet werden, dass wir sehr strikte Kriterien für die Klassifizierung benutzen. Menschen, die wir mit unserer Methode als sozial isoliert einstufen, haben einen sehr deutlichen Mangel im Austausch mit anderen. Personen, für die wir das Kriterium, einsam zu sein, als erfüllt ansehen, verspüren eine tiefe Einsamkeit.

Zentrale erklärende Variablen sind das Alter zum jeweiligen Befragungszeitpunkt und das Geschlecht. Zur Bestimmung des linearen Alterseffekts verwenden wir das Alter in Jahren, um mögliche nicht-lineare Alterseinflüsse zu erfassen, verwenden wir ergänzend das quadrierte Alter. Um feststellen zu können, ob Alters- und Geschlechterunterschiede in den Risikoverläufen für soziale Isolation und Einsamkeit einem gesellschaftlichen Wandel unterliegen, testen wir auch den Unterschied zwischen Geburtskohorten. Für die Untersuchung von Kohortenunterschieden beschränken wir uns auf Geburtsjahrgänge, für die im Deutschen Alterssurvey mehrere Messzeitpunkte über eine größere Altersspanne vorliegen, und bilden die folgenden drei Kohorten: Geburtsjahrgänge 1930-1939, 1940-1949, 1950-1959.

\section{Statistisches Analyseverfahren}

Für die Alternsverläufe schätzen wir Random-Effects-Panelregressionen. Dieses Verfahren fokussiert auf die individuellen Entwicklungen über die Messzeitpunkte und analysiert damit Alternsverläufe des Risikos sozialer Isolation und Einsamkeit.
Die Schätzungen erfolgen mittels logistischer Regressionsverfahren, da die abhängigen Variablen dichotom sind (isoliert vs. nicht isoliert; einsam vs. nicht einsam). Zur Bestimmung möglicher Unterschiede zwischen Frauen und Männern wird das Geschlecht sowohl als eigenständiger Prädiktor als auch als eine den Alternsverlauf moderierende Variable aufgenommen (Interaktion von Alter und Geschlecht). Die zusätzlichen Modelle zur Analyse möglicher Kohorteneffekte basieren auf den 1930 bis 1959 Geborenen und enthalten die drei 10-Jahres-Kohorten als Prädiktoren. Bei allen Modellierungen wurde zudem die regionale Zugehörigkeit zu den alten oder neuen Bundesländern als Kontrollvariable aufgenommen, um Ergebnisverzerrungen durch den überproportionalen Einbezug von in Ostdeutschland Wohnenden bei der Stichprobenziehung zu verhindern.

Sofern die im jeweiligen Regressionsmodell einbezogenen Prädiktoren einen statistisch signifikanten Einfluss $(p<0,05)$ auf das Isolations- oder Einsamkeitsrisiko haben, werden auf der Grundlage der Modellergebnisse altersjahrspezifische Wahrscheinlichkeitswerte für das Vorhandensein von Isolation oder Einsamkeit der Frauen und Männer berechnet. Diese lassen sich grafisch darstellen mit einem Wertebereich von null bis 100 Prozent. Die in den Abbildungen dargestellten Alternsverläufe kennzeichnen die sich aus den Alterseffekten vorhergesagten Wahrscheinlichkeiten von sozialer Isolation oder Einsamkeit. Sie beziffern das längsschnittliche Risiko, mit dem Älterwerden sozial isoliert oder einsam zu sein. In allen statistischen Untersuchungen wurden Unterschiede sowohl im Niveau bei verschiedenen Altersjahren als auch im linearen und quadratischen Alternsverlauf der Wahrscheinlichkeit von sozialer Isolation und Einsamkeit auf statistische Signifikanz geprüft.

Zunächst untersuchen wir, wie sich diese Risiken über die zweite Lebenshälfte für Frauen und Männer entwickeln und stellen hierzu den Risiko-

le“. Die Antworten auf die drei negativen Items werden umgepolt, so dass bei allen Items hohe Werte auf ein hohes Ausmaß von Einsamkeit verweisen. 
verlauf im Altersbereich von 40 bis 90 Jahren dar. Im nächsten Analyseschritt widmen wir uns den geschlechtsspezifischen Verläufen im Vergleich der
Geburtskohorten, die zwischen 1930 bis 1939, zwischen 1940 bis 1949 und zwischen 1950 bis 1959 geboren sind.

\subsection{Ergebnisse}

\subsubsection{Soziale Isolation im Verlauf der zweiten Lebenshälfte}

Das Risiko sozialer Isolation steigt mit dem Älterwerden. Bis etwa zum 75. Lebensjahr haben Männer ein höheres Isolationsrisiko als Frauen.

Im Folgenden berichten wir, wie sich das individuelle Älterwerden auf das Risiko, sozial isoliert und/oder einsam zu sein, bei Frauen und Männern auswirkt. Insgesamt zeigt sich ein über die zweite Lebenshälfte ansteigendes Risiko sozialer Isolation mit einem signifikanten Geschlechterunterschied im Alternsverlauf. Wie in Abbildung 5-1 dargestellt, steigt das Risiko sozial isoliert zu sein, mit dem Älterwerden an. Im Alter von 40 Jahren liegt dieses Risiko für beide Geschlechter bei etwa vier Prozent. Im Verlauf des weiteren Lebens steigt das Isolationsrisiko im Durchschnitt auf etwa 22 Prozent im Alter von 90 Jahren an.

Für Frauen und Männer gestalten sich diese Verläufe jedoch unterschiedlich. Männer weisen im Alter von 40 Jahren ein Isolationsrisiko von etwa fünf Prozent auf und dieses Risiko steigt ste-

Abbildung 5-1 Risiko sozialer Isolation bei Frauen und Männern im Alternsverlauf

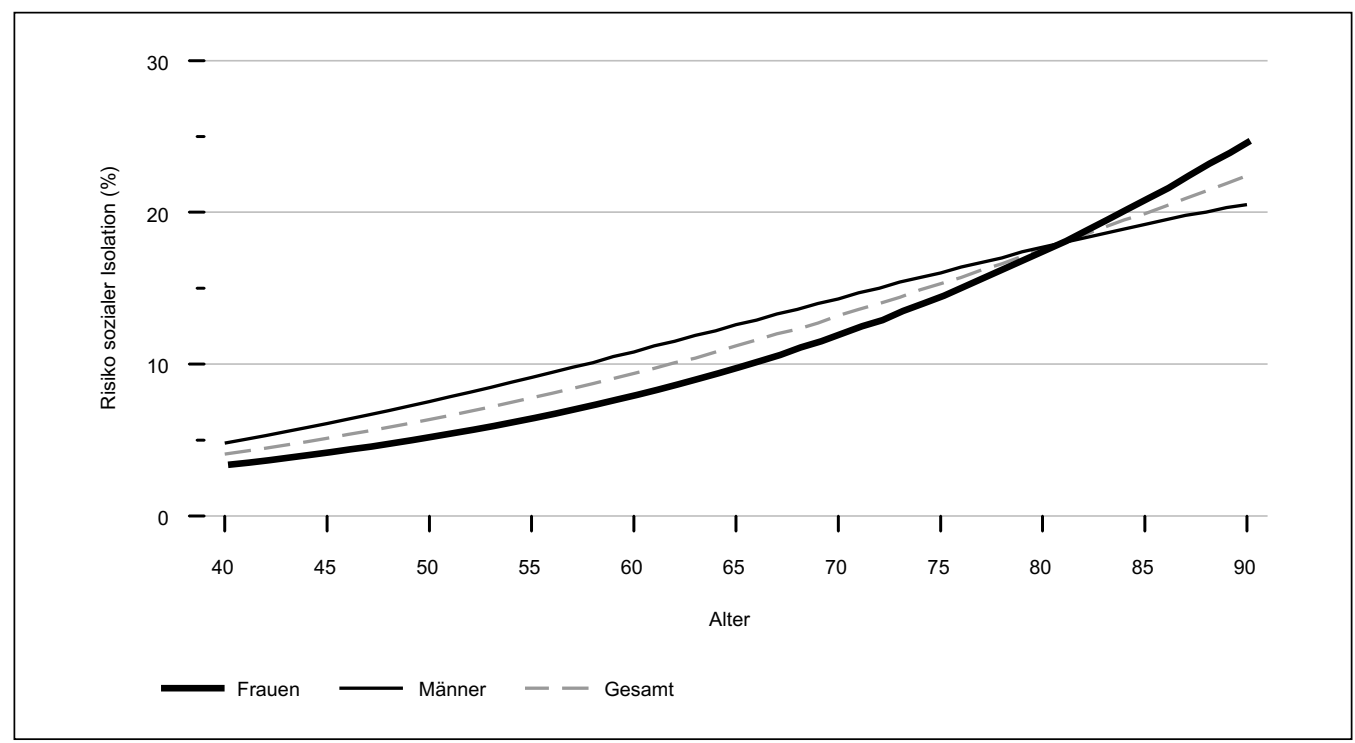

Quelle: DEAS 1996-2017, n = 19969 Studienteilnehmende mit insgesamt n = 39186 Beobachtungen; modellbasierte Schätzungen kontrolliert für Region; der Geschlechterunterschied im Alternsverlauf ist signifikant.

Die Wahrscheinlichkeit, im Alter von 65 Jahren sozial isoliert zu sein, beträgt für Frauen ca. zehn Prozent und für Männer ca. 13 Prozent. 
tig auf etwa 20 Prozent im Alter von 90 Jahren an. Das Risiko, isoliert zu sein, liegt bei Frauen Anfang 40 bei etwa drei Prozent. Dieses Risiko steigt zunächst weniger stark an als bei den Männern, erfährt aber im späteren Lebensverlauf etwa ab Ende 60 einen beschleunigten Zuwachs. Dementsprechend unterscheidet sich das Risiko, sozial isoliert zu sein, im mittleren Lebensalter und frühen hohen Alter zwischen Männern und Frauen. Im Alter von 65 Jahren haben Frauen beispielsweise ein Risiko von etwa zehn Prozent während Männer ein Risiko von etwa 13 Prozent aufweisen. Im hohen Alter gleicht sich das Risiko wieder an. Die Geschlechter unterscheiden sich ab dem 75 . Lebensjahr nicht mehr signifikant.

Der Alternsverlauf des Risikos, sozial isoliert zu sein, hat sich gewandelt. Bei später geborenen Geburtskohorten steigt das Isolationsrisiko mit dem Älterwerden nicht mehr auf einen so hohen Wert an wie bei den früher geborenen Jahrgängen.
Es zeigten sich signifikante Unterschiede in den geschätzten Risikoverläufen sozialer Isolation zwischen den zusammengefassten Geburtsjahrgängen von 1930-1939, 1940-1949 und 1950-1959 (Abbildung 5-2). Im Untersuchungszeitraum haben sich die altersspezifischen Risiken des Auftretens sozialer Isolation zwischen den drei betrachteten Kohorten eher verringert. Diese Verringerung wird sich aller Voraussicht nach insbesondere nach dem Erreichen des 60. Lebensjahres besonders bemerkbar machen. Während die Schätzung des allgemeinen Verlaufs unter Einschluss aller Geburtsjahrgänge (1911 bis 1974) einen stetigen Zuwachs des Isolationsrisikos über das Lebensalter anzeigt, so ergibt die vergleichende Betrachtung der drei Geburtskohorten 1930 bis 1939, 1940 bis 1949 und 1950 bis 1959 ein anderes Bild. Bei den zwischen 1930 und 1939 Geborenen steigt das Isolationsrisiko bis zum Alter Mitte 70 und stabilisiert sich dann bis in höchste Alter bei etwa 16 Prozent. Für die nachfolgenden Geburtskohorten zeigt sich die Abflachung

\section{Abbildung 5-2 Risiko sozialer Isolation nach Kohorten im Alternsverlauf}

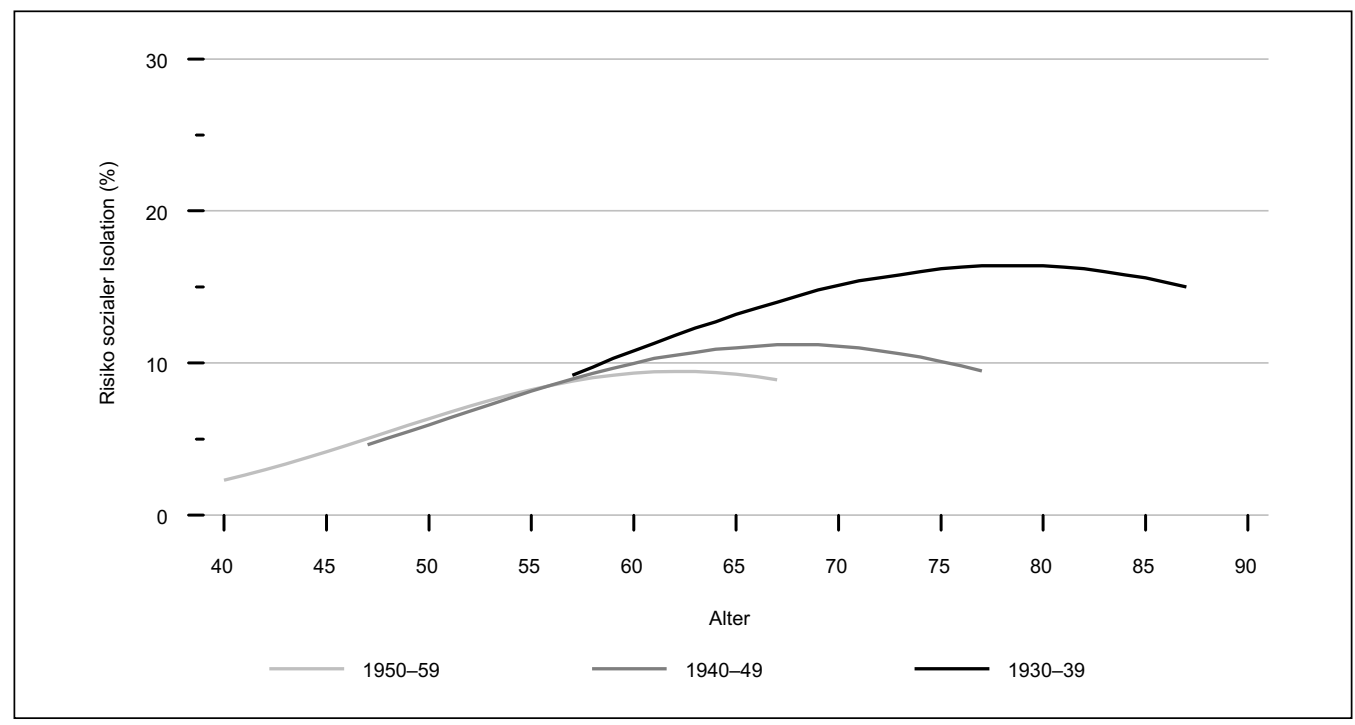

Quelle: DEAS 1996-2017, n = 13899 Studienteilnehmende mit insgesamt n = 29069 Beobachtungen; modellbasierte Schätzungen kontrolliert für Region und Geschlecht; die Kohortenunterschiede sind signifikant.

Die Wahrscheinlichkeit, im Alter von 65 Jahren sozial isoliert zu sein, beträgt für die 1930-1939 Geborenen ca. 13 Prozent und für die 1950-1959 Geborenen ca. neun Prozent. 
des Isolationsrisikos in noch früherem Alter und auf einem tieferen Niveau. Bei den Geburtsjahrgängen zwischen 1940 und 1949 endet der alternsbezogene Anstieg des Isolationsrisikos schon etwa beim 70. Lebensjahr und das Risiko erreicht nur noch ein maximales Niveau von etwa elf Prozent. Für die Jahrgänge 1950 bis 1959 endet der Anstieg schon beim Alter von 65 Jahren mit einem maximalen Isolationsrisiko von nur noch neun Prozent.

Für alle Kohorten zeigen sich ähnliche Geschlechterunterschiede in den Alternsverläufen des Isolationsrisikos.

Wie in Abbildung 5-3 dargestellt, werden die Geschlechterunterschiede im Vergleich über die Geburtskohorten weder kleiner noch größer; sie entsprechen in etwa den Geschlechterunterschieden, die in der Analyse aller Geburtsjahrgänge sichtbar wurde (vgl. Abbildung 5-1).

\subsubsection{Einsamkeit im Verlauf der zweiten Lebenshälfte}

Der Verlauf des Einsamkeitsrisikos in der zweiten Lebenshälfte ist u-förmig. Männer haben ein höheres Risiko im mittleren Lebensalter, Frauen haben ein höheres Risiko im hohen Alter.

Der Verlauf im Einsamkeitsrisiko von Frauen und Männern unterscheidet sich deutlich von der Entwicklung des Isolationsrisikos. Das Einsamkeitsrisiko ist zu Beginn der zweiten Lebenshälfte bei beiden Geschlechtern höher als das Isolationsrisiko, nimmt dann im Alternsverlauf bei Frauen und Männern zunächst ab, um im höheren Alter wieder anzusteigen (Abbildung 5-4). Während das Isolationsrisiko mit steigendem Alter relativ kontinuierlich zunimmt, zeigt sich bei der Einsamkeit ein signifikanter kurvilinearer Verlauf. 40-Jährige haben ein Einsamkeitsrisiko von elf Prozent. Diese Wahrscheinlichkeit verringert sich zunächst über die nächsten 30 Lebensjahre. Das Einsamkeitsrisiko im Alter von 70 Jahren liegt bei acht Prozent.
Danach steigt es wieder an. 90-jährige Menschen haben - wie die 40-Jährigen - ein Einsamkeitsrisiko von elf Prozent.

Im Alternsverlauf des Einsamkeitsrisikos gibt es einen signifikanten Geschlechterunterschied. Männer haben im mittleren Erwachsenenalter ein höheres Einsamkeitsrisiko als Frauen. Der Geschlechterunterschied nimmt mit steigendem Alter jedoch ab und dreht sich im Verlauf des Rentenalters um, so dass im hohen Alter mehr Frauen als Männer einsam sind. Im Alter von 40 Jahren beträgt das Risiko, einsam zu sein für Männer etwa 13 Prozent und für Frauen ca. zehn Prozent. Im weiteren Alternsverlauf verringert sich dieser Unterschied und gleicht sich etwa ab dem 62. Lebensjahr aus. Mit 65 Jahren haben Frauen und Männer ein nahezu gleich hohes Einsamkeitsrisiko von knapp acht Prozent. Danach steigt das Einsamkeitsrisiko mit dem Älterwerden stärker bei Frauen als bei Männern. Mit 90 Jahren haben Frauen ein Risiko von 14 Prozent, einsam zu sein. Bei Männern in diesem Alter beträgt das Einsamkeitsrisiko hingegen nur neun Prozent.

Bei später geborenen Geburtskohorten ist der Verlauf des Einsamkeitsrisikos weniger vom Lebensalter abhängig.

Es zeigen sich ebenfalls signifikante Unterschiede in den geschätzten Risikoverläufen zwischen den Geburtskohorten von 1930 bis 1939, 1940 bis 1949 und 1950 bis 1959 (Abbildung 5-5). Im Untersuchungszeitraum haben sich die altersspezifischen Risiken des Auftretens von Einsamkeit zwischen den drei betrachteten Kohorten tendenziell verringert. Die Veränderungen über die Geburtskohorten sind allerdings schwerer zu erkennen als die Kohortenunterschiede in der sozialen Isolation. Abbildung 5-5 zeigt, dass das Einsamkeitsniveau im Alter zwischen 40 und 50 Jahren bei der jüngsten der drei betrachteten Kohorten gesunken ist und sich im Alternsverlauf bis Mitte 60 wenig verändert. Im Vergleich zur ältesten betrachteten Kohorte deutet sich eine Verlagerung des altersbezogenen Anstiegs des Einsamkeitsrisi- 
Abbildung 5-3 Risiko sozialer Isolation bei Frauen und Männern nach Kohorten im Alternsverlauf

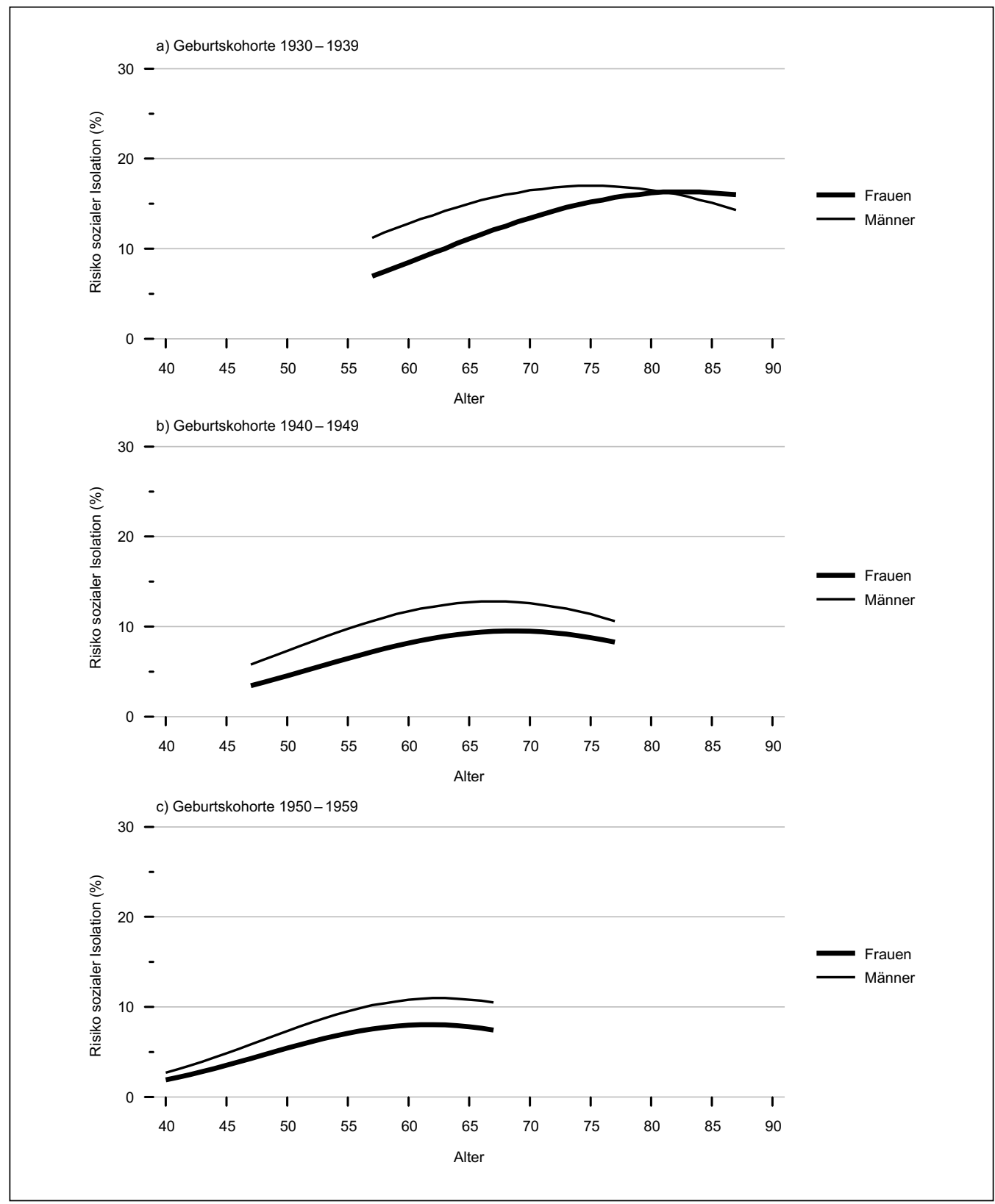

Quelle: DEAS 1996-2017, n = 13899 Studienteilnehmende mit insgesamt $n=29069$ Beobachtungen; modellbasierte Schätzungen kontrolliert für Region; kein signifikanter Interaktionseffekt zwischen Geschlecht und Geburtskohorte, die Geschlechterunterschiede haben sich zwischen den Geburtskohorten nicht verändert.

Die Wahrscheinlichkeit, im Alter von 65 Jahren sozial isoliert zu sein, beträgt für 1950-1959 geborene Männer ca. elf Prozent, für Frauen ca. acht Prozent. 
Abbildung 5-4 Einsamkeitsrisiko bei Frauen und Männern im Alternsverlauf

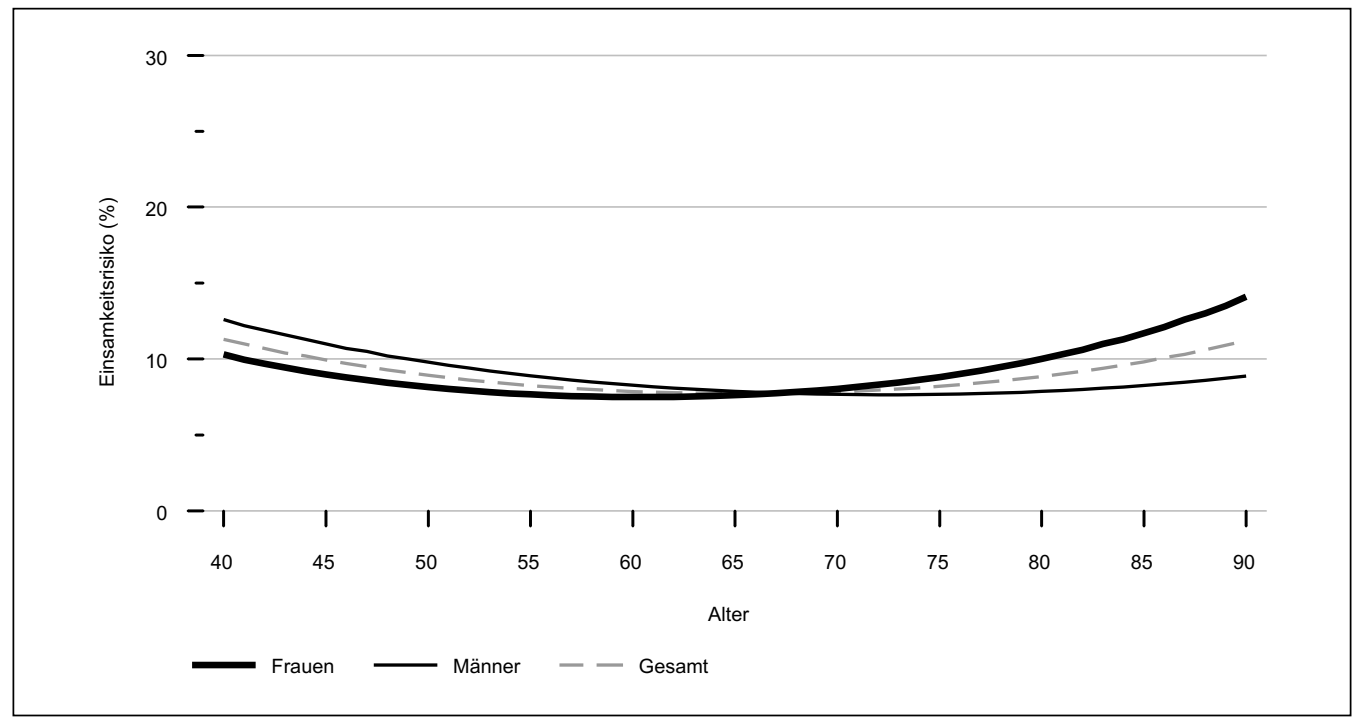

Quelle: DEAS 1996-2017, n = 16151 Studienteilnehmende mit insgesamt $n=31532$ Beobachtungen; modellbasierte Schätzungen kontrolliert für Region; der Geschlechtereffekt ist signifikant.

Die Wahrscheinlichkeit, im Alter von 90 Jahren einsam zu sein, beträgt für Frauen ca. 14 Prozent und für Männer ca. neun Prozent.

\section{Abbildung 5-5 Einsamkeitsrisiko nach Kohorten im Alternsverlauf}

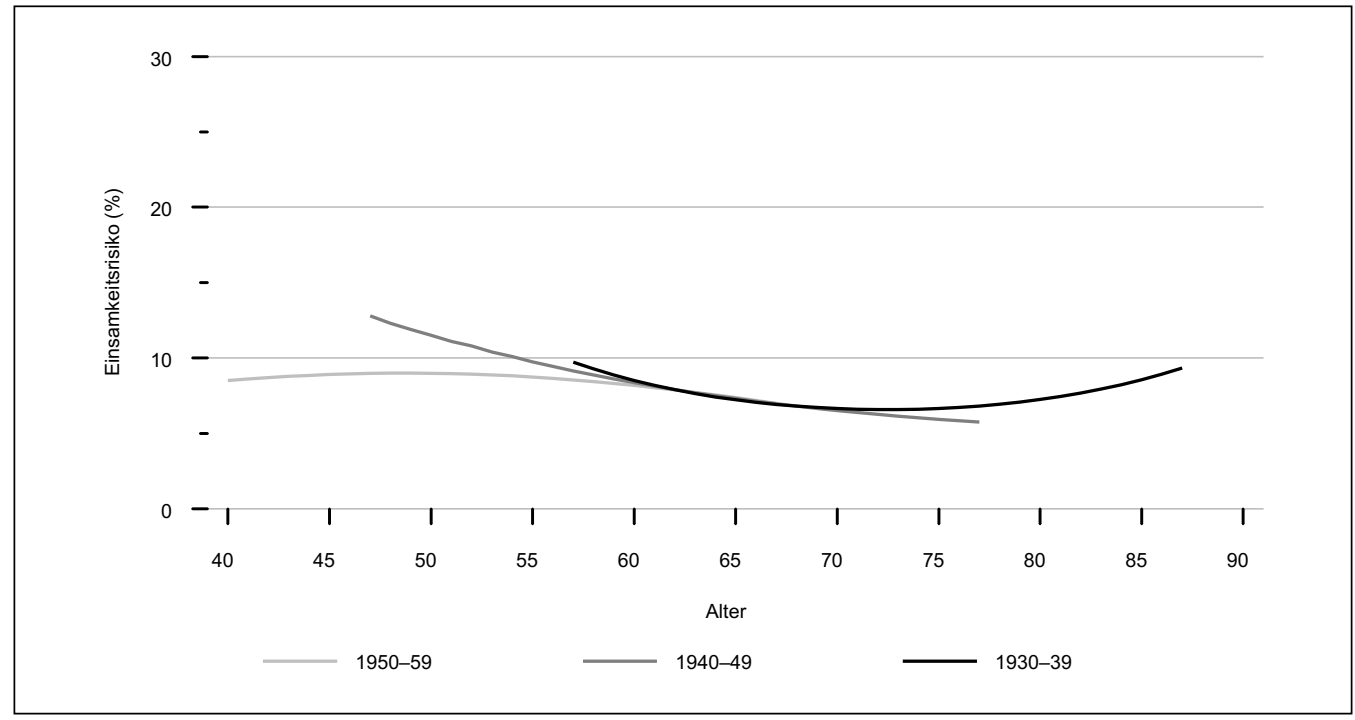

Quelle: DEAS 1996-2017, n = 11405 Studienteilnehmende mit insgesamt n = 23397 Beobachtungen; modellbasierte Schätzungen kontrolliert für Region und Geschlecht; der Kohorteneffekt ist signifikant.

Alle drei Geburtsjahrgangsgruppen haben im Alter von 65 Jahren ein Einsamkeitsrisiko von ca. sieben Prozent. 
kos in höhere Altersjahre an. Mit anderen Worten: Das Einsamkeitsrisiko ist in den später geborenen Jahrgängen weniger stark mit dem Älterwerden verknüpft als in den früher geborenen Geburtskohorten.

In allen betrachteten Kohorten zeigen sich ähnliche Geschlechterunterschiede in den Alternsverläufen des Einsamkeitsrisikos.

Wie beschrieben gibt es Unterschiede in den Alternsverläufen des Einsamkeitsrisikos sowohl zwischen Frauen und Männern als auch zwischen Geburtsjahrgängen. Deshalb wurde in einem nächsten Untersuchungsschritt betrachtet, ob oder inwieweit sich die gleichen Geschlechterunterschiede in den verschiedenen Geburtskohorten wiederfinden lassen. $\mathrm{Zu}$ diesem Zweck wurde untersucht, ob sich statistisch eine Interaktion zwischen den Faktoren Geschlecht und Kohorte in Bezug auf die Alternsverläufe nachweisen ließ.

\subsection{Diskussion}

\section{Zusammenfassung}

Unsere längsschnittlichen Analysen der individuellen Entwicklungen zeigen, dass das Risiko sozialer Isolation zu Beginn der zweiten Lebenshälfte noch sehr niedrig ist, aber mit dem Älterwerden steigt und sich bei Frauen und Männern unterschiedlich entwickelt. Männer im Alter von 40 Jahren haben ein etwas höheres Isolationsrisiko als Frauen. Zudem steigt dieses im Verlauf der zweiten Lebenshälfte relativ gleichmäßig bis ins hohe Alter an. Bei Frauen ist das Isolationsrisiko anfangs niedriger und nimmt zunächst auch weniger stark zu. Im Rentenalter erhöht sich das Isolationsrisiko der Frauen jedoch schneller als das der Männer, so dass sie im hohen Alter (80 Jahre und älter) ähnlich häufig oder sogar etwas häufiger dem Risiko ausgesetzt sind, sozial isoliert zu sein. Zuvor haben
Die statistische Analyse ergab keinen signifikanten Interaktionseffekt von Geschlecht und Geburtskohorte, das heißt die Geschlechterunterschiede der Verläufe des Einsamkeitsrisikos haben sich nicht verändert. Berücksichtigt man den Interaktionsterm dennoch in der modellbasierten Schätzung der Alternsverläufe des Einsamkeitsrisikos, ergibt sich folgendes Bild (Abbildung 5-6). In der jüngsten der drei Kohorten zeigt sich das bereits erwähnte höhere Einsamkeitsrisiko der Männer zwischen dem 40. und 60. Lebensjahr, die mittlere Kohorte weist geringe Geschlechterdifferenzen auf und in der ältesten der drei Kohorten zeichnet sich der stärkere Risikoanstieg bei den Frauen im hohen Alter ab. Dass der Geschlechterunterschied im hohen Alter in Abbildung 5-6 nicht so deutlich hervortritt wie in der Gesamtschau in Abbildung 5-4 dürfte der Tatsache geschuldet sein, dass in Abbildung 5-4 alle Untersuchungsteilnehmerinnen und -teilnehmer, auch die vor $1930 \mathrm{Ge}$ borenen einbezogen sind, die besonders die Befunde zum höheren Alter beeinflussen.

Frauen allerdings mehr als drei Lebensjahrzehnte lang von Anfang 40 bis Mitte 70 ein geringeres Isolationsrisiko als Männer. In der Abfolge der Geburtskohorten verändert sich die Entwicklung des Isolationsrisikos mit dem Älterwerden. In später geborenen Geburtskohorten wird das Isolationsrisiko im höchsten Lebensalter vermutlich nicht mehr weiter ansteigen und unter dem früher geborener Geburtsjahrgänge bleiben. Die Geschlechterunterschiede in den Alternsverläufen sozialer Isolation sind weitgehend stabil geblieben und haben sich in der Abfolge der untersuchten Kohorten weder verringert noch vergrößert.

Das Einsamkeitsrisiko weist im Alternsverlauf von 40 bis 90 Jahren einen leicht u-förmigen Verlauf auf. Im Beobachtungszeitraum ist es am höchsten im Alter von 40 Jahren und liegt in diesem Alter deutlich über dem Isolationsrisiko. Im 
Abbildung 5-6 Einsamkeitsrisiko bei Frauen und Männern nach Kohorten im Alternsverlauf

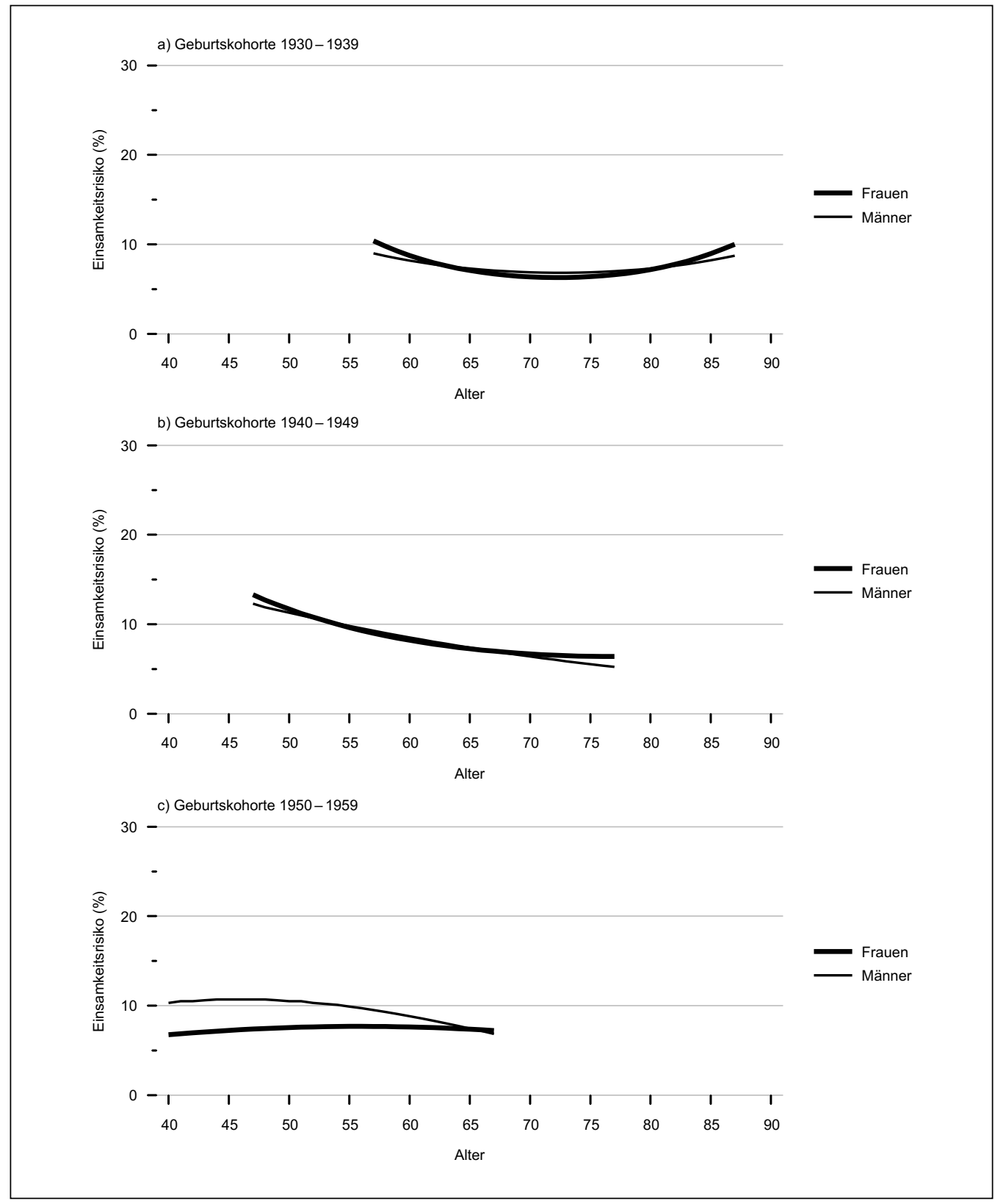

Quelle: DEAS 1996-2017, n = 11405 Studienteilnehmende mit insgesamt n = 23397 Beobachtungen; modellbasierte Schätzungen kontrolliert für Region; kein signifikanter Interaktionseffekt zwischen Geschlecht und Geburtskohorte, die Geschlechterunterschiede haben sich zwischen den Geburtskohorten nicht verändert.

Die Wahrscheinlichkeit, im Alter von 6o Jahren einsam zu sein, beträgt für 1950-1959 geborene Männer ca. neun Prozent und für Frauen ca. acht Prozent. 
weiteren Alternsverlauf verringert es sich bis etwa zum 70. Lebensjahr und steigt dann langsam wieder an. Wenn Menschen 90 Jahre alt sind, haben sie in etwa das gleiche Einsamkeitsrisiko wie mit 40 Jahren. Die Form des Alternsverlaufs im Einsamkeitsrisiko unterscheidet sich zwischen den Geschlechtern und Geburtskohorten. Während Männer von 40 bis etwa 60 Jahren ein vergleichsweise höheres Einsamkeitsrisiko erleben, haben Frauen ein größeres Einsamkeitsrisiko als Männer, nachdem sie etwa das 70. Lebensjahr erreicht haben. Bei später geborenen Geburtsjahrgängen zeigt sich ein etwas niedrigeres Einsamkeitsrisiko zu Beginn der zweiten Lebenshälfte und eine Abflachung des u-förmigen Verlaufs. Aller Voraussicht nach wird sich der Anstieg des Einsamkeitsrisikos im hohen Alter abschwächen. Die Unterschiede in den Alternsverläufen zwischen den Geschlechtern sind von diesem sozialen Wandel nicht betroffen.

\section{Interpretation}

Wenn man sich die Verläufe über die gesamte zweite Lebensspanne genauer ansieht, kristallisieren sich bestimmte Lebensphasen heraus, in denen älterwerdende Menschen besonders verwundbar zu sein scheinen. Das Risiko sozial isoliert zu sein, erreicht im hohen Erwachsenenalter die höchsten Werte. Wie aus der Forschung bekannt ist, verringert sich die Größe der sozialen Netzwerke kontinuierlich über die zweite Lebenshälfte (Wrzus, Hanel, Wagner, \& Neyer 2013). Wie unsere Analysen unseres Wissens nach erstmalig zeigen, korrespondiert diese allgemeine Entwicklung mit einer in weiten Teilen linearen Zunahme des individuellen Risikos sozialer Isolation im Verlauf der zweiten Lebenshälfte. Die Verringerung der Netzwerkgröße führt demnach häufig dazu, dass die betroffenen Menschen niemanden mehr oder nur noch eine einzige Person haben, mit der sie regelmäßig Kontakt haben und die für sie wichtig ist. Diese negative Entwicklung im Alter kann unter anderem bedingt sein durch den Verlust von Netzwerkpersonen durch Mortalität oder Wegzug, aber auch durch schwindende gesundheitliche Ressourcen, die bedeutsame soziale Kontakte erschweren (Huxhold, Fiori, \& Windsor 2013; Huxhold \& Fiori 2018).

Frauen und Männer unterscheiden sich in ihren Risikolagen im Lebensverlauf. Im mittleren Bereich der beobachteten Lebensspanne etwa ab dem Alter von 40 Jahren bis hin zum 75 . Lebensjahr haben Frauen ein geringeres Risiko als Männer sozial isoliert zu sein. Dieser Vorteil hat möglicherweise mit einem höheren Investment von Frauen in ihre sozialen Netzwerke, insbesondere in ihre Freundschaften, zu tun. Erst im hohen Erwachsenenalter nach dem 75 . Lebensjahr scheinen die verstärkt bei Frauen vorkommenden funktionalen Einschränkungen (siehe Kapitel 3 Funktionale und subjektive Gesundheit in diesem Band) und das vergleichsweise hohe weibliche Verwitwungsrisiko den sozialen Integrationsvorteil von Frauen aufzuheben.

Insgesamt lassen die Ergebnisse jedoch erkennen, dass selbst im Alter von 90 Jahren rund 80 Prozent der in einem Privathaushalt lebenden Menschen in Deutschland vermutlich nicht isoliert sein werden. Die Mehrheit der Hochbetagten pflegt mindestens zwei Beziehungen zu anderen ihnen wichtigen Personen mit regelmäßigem Austausch. Das mit 20 Prozent dennoch relativ hohe Risiko sozialer Isolation im hohen Alter schlägt sich nicht im besonderen Maße in einem proportional gestiegenen Einsamkeitsrisiko nieder.

Obwohl unsere Untersuchungen einen leichten Anstieg des Einsamkeitsrisikos ab etwa dem 70. Lebensjahr verzeichnen, bewegt sich das Einsamkeitsrisiko beim Erreichen des 90. Lebensjahres auf dem gleichen Niveau wie das Risiko im Alter von 40 Jahren. Dieses Phänomen lässt sich möglicherweise dadurch erklären, dass sich mit dem Älterwerden die individuellen Bedürfnisse in Bezug auf soziale Kontakte ändern und sich Menschen im hohen Alter insbesondere auf den emotionalen Austausch konzentrieren (Carstensen, Isaacowitz, \& Charles 1999). Dieses emotionale Bedürfnis kann möglicherweise auch von sehr wenigen, vielleicht sogar von nur einer Person befriedigt werden. 
Ein starkes Bedürfnis nach sozialer Integration könnte hingegen erklären, warum unsere Analysen ein vergleichsweise hohes Risiko für Einsamkeit zwischen dem 40. und dem 50. Lebensjahr verzeichnen. In der Literatur wird häufig angenommen, dass gerade diese Lebensphase gekennzeichnet ist durch intensive Prozesse der Selbstbewertung, in denen die persönlichen Errungenschaften in allen Lebensbereichen, auch im Bereich der sozialen Beziehungen, sowohl mit den eigenen Erwartungen als auch den vorherrschenden sozialen Normen verglichen werden (Freund \& Ritter 2009). Deshalb mögen negative kritische Lebensereignisse - wie die Auflösung einer Partnerschaft oder ein Krankheitsereignis - zu diesem Zeitpunkt besonders starke Auswirkungen auf das Risiko haben einsam zu werden, denn die eigenen und sozialen Erwartungen sind in dieser Lebensphase auf Grund der oben genannten Vergleichsprozesse besonders präsent.

Die Einsamkeitsrisikoverläufe von Frauen und Männern sind unterschiedlich und spiegeln in gewisser Weise die Geschlechterunterschiede in den Verläufen des Isolationsrisikos wider. Im Alter von 40 bis 60 Jahren haben Männer ein höheres Risiko als Frauen einsam zu sein, vermutlich weil Frauen in dieser Zeit ihre sozialen Beziehungen intensiver pflegen. Mit steigendem Alter haben Frauen eine höhere Wahrscheinlichkeit als Männer, von Verwitwung und Mobilitätseinschränkungen betroffen zu sein. Diese geschlechtsspezifischen Risikofaktoren führen vermutlich dazu, dass das Einsamkeitsrisiko ab Anfang 70 für Frauen stärker ansteigt als für Männer.

Angesichts der öffentlichen Debatte um eine vermeintliche „Epidemie der Einsamkeit“ ist es wichtig, ein Ergebnis besonders hervorzuheben: In unseren Analysen der individuellen Verläufe der Risiken für soziale Isolation und Einsamkeit von Männern und Frauen ergaben sich Anzeichen für eine Abschwächung der altersbezogenen Zunahme des Einsamkeits- und Isolationsrisikos und für eine Stagnation des Anteils der im Alter isoliert lebenden und/oder sich einsam fühlenden Menschen. Anders ausgedrückt: Wenn sich die gesell- schaftlichen Gegebenheiten in der nächsten Dekade nicht radikal ändern, werden die dann 70- bis 90-Jährigen nicht häufiger isoliert oder einsam sein als die heute 70- bis 90-Jährigen. Wahrscheinlich werden die oft vermuteten negativen Auswirkungen auf die sozialen Beziehungen der häufig berichteten Trends zu einer vermehrten Kinderlosigkeit, erhöhten Trennungsraten und größeren räumlichen Wohnentfernungen zwischen Eltern und ihren erwachsenen Kindern ausgeglichen durch eine größere Wichtigkeit von Freundschaften und verbesserte Kommunikationsmöglichkeiten. Auswertungen des Deutschen Alterssurveys (DEAS) weisen beispielsweise auf eine in den letzten Jahrzehnten steigende Bedeutung von Freundinnen und Freunden im persönlichen Netzwerk hin (Huxhold et al. 2010). Der gesellschaftliche Wandel in den Familienbeziehungen über die verschiedenen Geburtskohorten hinweg und die Entwicklung zu mehr Freiheit in der Gestaltung der sozialen Beziehungen (Allan 2008) führen demnach zwar zu einer Veränderung in der Zusammensetzung der persönlichen Netzwerke, aber vermutlich nicht zu einer problematischen Verringerung der persönlichen Netzwerkgrößen oder einer Zunahme an Einsamkeit und sozialer Isolation.

\section{Politische Implikationen}

Die Analysen, die in diesem Kapitel präsentiert wurden, können zwei wichtige Erkenntnisse zu der öffentlichen Debatte um eine sich vermeintlich ausbreitende Einsamkeit („Epidemie“) beitragen. Soziale Isolation und Einsamkeit sind keine unausweichlichen Schicksale des Alter(n)s. Die überwiegende Mehrheit aller Menschen wird auch im hohen Alter weder sozial isoliert noch einsam sein. Außerdem hat der Anteil an Frauen und Männern, die soziale Isolation und Einsamkeit im Alter erleben, nicht zugenommen und wird es aller Voraussicht nach auch in der nächsten Dekade nicht. Diese Erkenntnisse sind deshalb so wichtig, weil die Erwartungen, die Menschen von ihrem eigenen Alter haben, zu selbsterfüllenden Prophezeiungen 
werden können, die den eigenen Alternsprozess beeinflussen (Levy 2009). Eine neuere Studie fand in diesem Zusammenhang heraus, dass gerade Menschen mit einem positiven Ausblick auf das eigene Älterwerden besser in der Lage sind, Freundschaften aufrechtzuerhalten und im Alter neue zu schließen (Menkin, Robles, Gruenewald, Tanner, \& Seeman 2017). Zusätzlich ergaben weitere Analysen auf Grundlage des Deutschen Alterssurveys, dass ein Teil des Trends zur wachsenden Bedeutung und Wichtigkeit von Freundschaften im höheren Erwachsenenalter mit einer Verbesserung der Vorstellungen vom Älterwerden zusammenhängt (Huxhold 2019). Eine einseitige öffentliche Debatte über Einsamkeit, die auf einer Unausweichlichkeit sozialer Isolation und Einsamkeit im Alter beharrt, hat demzufolge das Potenzial, die individuellen Vorstellungen vom Älterwerden zum Negativen zu beeinflussen, und ruft vielleicht dadurch erst das Phänomen hervor, das es eigentlich $\mathrm{zu}$ vermeiden versucht.

Dennoch ist die öffentliche Debatte um das Thema wichtig. Trotz der eher erfreulichen Gesamtergebnisse, die in diesem Kapitel präsentiert wurden, muss man bedenken, dass unsere Kriterien für soziale Isolation und Einsamkeit sehr strikt sind. Das bedeutet, dass Menschen, die gemäß unserer Klassifikation als sozial isoliert oder einsam eingestuft werden, sich in stark emotional belastenden und Beziehungsressourcen einschränkenden Situationen befinden, die mit einer ganzen Reihe von Risiken für die mentale und physische
Gesundheit einhergehen. Obwohl die überwiegende Mehrheit der Frauen und Männer weder sozial isoliert noch einsam sind, werden immer noch mehrere hunderttausend Menschen in der zweiten Lebenshälfte von sozialer Isolation und Einsamkeit betroffen sein. Angesichts der schwerwiegenden gesundheitlichen Folgen bedeutet deshalb die Bekämpfung sozialer Isolation und Einsamkeit nichts anderes als breitflächige Gesundheitsvorsorge.

Ein erster Schritt dazu könnte eine Aufklärungskampagne sein, die das soziale Stigma bekämpft, welches es vielen Menschen - Frauen wie Männern - schwer macht, über ihre Einsamkeit zu reden und sich Hilfe zu holen (de Jong Gierveld, van Tilburg, \& Dykstra 2006). Eine solche Aufklärungskampagne muss so gestaltet sein, dass sich Frauen und Männer gleichermaßen angesprochen fühlen. Und es muss mit Augenmaß vorgegangen werden, um kein Stereotyp vom einsamen Alter zu bedienen und eine Verschlechterung der Vorstellungen vom Älterwerden zu vermeiden. Im nächsten Schritt sollten Maßnahmen ergriffen werden, die es mehr Menschen als bisher ermöglichen, sich aus ihrer Isolation oder Einsamkeit zu befreien. Dazu gehören beispielsweise niedrigschwellige Interaktions- und Teilhabeangebote im örtlichen Nahraum wie die Förderung von Nachbarschaftskontakten. Basierend auf unseren Analysen könnten sich diese Interventionen besonders auf das jüngere mittlere Lebensalter und das hohe Alter konzentrieren.

\section{Literatur}

Allan, G. (2008). Flexibility, friendship, and family. Personal Relationships, 15(1), 1-16.

Antonucci, T. C., Lansford, J. E., Akiyama, H., Smith, J., Baltes, M. M., Takahashi, K., Fuhrer, R., \& Dartigues, J. F. (2002). Differences between men and women in social relations, resource deficits, and depressive symptomatology during later life in four nations. Journal of Social Issues, 58(4), 767-783. doi: 10.1111/1540-4560.00289.

Baumeister, R. F., \& Leary, M. R. (1995). The need to belong - desire for interpersonal attachment as a fundamental human-motivation. Psychological Bulletin, 117(3), 497-529. doi: 10.1037/0033-2909.11 7.3.497. 
Böger, A., \& Huxhold, O. (2018). Age-Related Changes in Emotional Qualities of the Social Network From Middle Adulthood Into Old Age: How Do They Relate to the Experience of Loneliness? Psychology and Aging, 33(3), 482-496. doi: 10.1037/pag0000222.

Böger, A., Wetzel, M., \& Huxhold, O. (2017). Allein unter vielen oder zusammen ausgeschlossen: Einsamkeit und wahrgenommene soziale Exklusion in der zweiten Lebenshälfte. In: K. Mahne, J. K. Wolff, J. Simonson \& C. Tesch-Römer (Hrsg.) Altern im Wandel. Zwei Jahrzehnte Deutscher Alterssurvey (DEAS) (S. 273-285). Wiesbaden: Springer VS.

Carstensen, L. L., Isaacowitz, D. M., \& Charles, S. T. (1999). Taking time seriously - A theory of socioemotional selectivity. American Psychologist, 54(3), 165-181. doi: 10.1037//0003-066x.54.3.165.

Drinkuth, K. (2018). Die Einsamkeit breitet sich in Deutschland aus wie eine Epidemie. Tagesspiegel Online, 05. 03. 2018. Verfügbar unter https://www.tagesspiegel.de/weltspiegel/soziale-isolation-dieeinsamkeit-breitet-sich-in-deutschland-aus-wie-eine-epidemie/21035520.html [abgerufen am 11.12. 2018]

Engstler, H., \& Tesch-Römer, C. (2010). Lebensformen und Partnerschaft. In: A. Motel-Klingebiel, S. Wurm \& C. Tesch-Römer (Hrsg.) Altern im Wandel. Befunde des Deutschen Alterssurveys (DEAS) (S. 163-187). Stuttgart: Kohlhammer.

Fischer, C. S., \& Beresford, L. (2015). Changes in Support Networks in Late Middle Age: The Extension of Gender and Educational Differences. The Journals of Gerontology, Series B: Psychological Sciences and Social Sciences, 70(1), 123-131. doi: 10.1093/geronb/gbu057.

Freund, A. M., \& Ritter, J. O. (2009). Midlife Crisis: A Debate. Gerontology, 55(5), 582-591. doi: 10.11 $59 / 000227322$.

Hawkley, L. C., \& Cacioppo, J. T. (2010). Loneliness Matters: A Theoretical and Empirical Review of Consequences and Mechanisms. Annals of Behavioral Medicine, 4O(2), 218-227.

Hawthorne, G. (2006). Measuring Social Isolation in Older Adults: Development and Initial Validation of the Friendship Scale. Social Indicators Research, 77(3), 521-548. doi: 10.1007/s11205-005-7746-y.

Huxhold, O. (2019, eingereichtes Manuskript). Gauging Effects of Historical Changes on Aging Trajectories: The Increasing Importance of Friendships.

Huxhold, O., \& Fiori, K. L. (2018). Do demographic changes jeopardize social integration among aging adults living in rural regions? The Journals of Gerontology, Series B: Psychological Sciences and Social Sciences, gby008-gby008. doi: 10.1093/geronb/gby008.

Huxhold, O., Fiori, K. L., \& Windsor, T. D. (2013). The dynamic interplay of social network characteristics, subjective well-being and health: The costs and benefits of socio-emotional selectivity. Psychology and Aging, 28(1), 3-16. doi: 10.1037/a0030170.

Huxhold, O., Mahne, K., \& Naumann, D. (2010). Soziale Integration. In: A. Motel-Klingebiel, S. Wurm \& C. Tesch-Roemer (Hrsg.) Altern im Wandel. Befunde des Deutschen Alterssurveys (DEAS) (S. 215-232). Stuttgart: Kohlhammer.

Jong Gierveld J. de, Tilburg, T. G. van, \& Dykstra, P. A. (2006). Loneliness and social isolation. In: A. Vangelisti \& D. Perlman (Hrsg.) Handbook of personal relationships (S. 485-500). Cambridge, England: Cambridge University Press.

Levy, B. (2009). Stereotype embodiment: A psychosocial approach to aging. Current Directions in Psychological Science, 18(6), 332-336. doi: 10.1111/j.1467-8721.2009.01662.x.

Luo, Y., Hawkley, L. C., Waite, L. J., \& Cacioppo, J. T. (2012). Loneliness, health, and mortality in old age: A national longitudinal study. Social Science \& Medicine, 74(6), 907-914. doi: 10.1016/j.socscimed.20 11.11.028. 
Mahne, K., \& Huxhold, O. (2017). Nähe auf Distanz: Bleiben die Beziehungen zwischen älteren Eltern und ihren erwachsenen Kindern trotz wachsender Wohnentfernungen gut? In: K. Mahne, J. K. Wolff, J. Simonson \& C. Tesch-Römer (Hrsg.) Altern im Wandel. Zwei Jahrzehnte Deutscher Alterssurvey (DEAS) (S. 215-230). Wiesbaden: Springer VS.

Menkin, J. A., Robles, T. F., Gruenewald, T. L., Tanner, E. K., \& Seeman, T. E. (2017). Positive Expectations Regarding Aging Linked to More New Friends in Later Life. The Journals of Gerontology, Series B: Psychological Sciences and Social Sciences, 72(5), 771-781. doi: 10.1093/geronb/gbv118.

Miche, M., Huxhold, O., \& Stevens, N. L. (2013). A latent class analysis of friendship network types and their predictors in the second half of life. The Journals of Gerontology, Series B: Psychological Sciences and Social Sciences, 68(4), 644-652. doi: 10.1093/geronb/gbt041.

Perlman, D., \& Peplau, L. A. (1981). Toward a social psychology of loneliness. In: R. Gilmour \& S. Duck (Hrsg.) Personal Relationships: 3. Relationships in Disorder (S. 31-56). London: Academic Press.

Perrig-Chiello, P., Spahni, S., Hopflinger, F., \& Carr, D. (2016). Cohort and Gender Differences in Psychosocial Adjustment to Later-Life Widowhood. The Journals of Gerontology, Series B: Psychological Sciences and Social Sciences, 71(4), 765-774. doi: 10.1093/geronb/gbv004.

Sander, J., Schupp, J., \& Richter, D. (2017). Getting Together: Social Contact Frequency Across the Life Span. Developmental Psychology, 53(8), 1571-1588. doi: 10.1037/dev0000349.

Soest T. von, Luhmann, M., Hansen, T., \& Gerstorf, D. (2018). Development of loneliness in midlife and old age: Its nature and correlates. Journal of Personality and Social Psychology, Online First Publication. doi: $10.1037 /$ pspp0000219.

Stevens, N. L., \& Tilburg, T. G. van (2011). Cohort differences in having and retaining friends in personal networks in later life. Journal of Social and Personal Relationships, 28(1), 24-43. doi: 10.1177/026540 7510386191.

Suanet, B., \& Antonucci, T. C. (2017). Cohort Differences in Received Social Support in Later Life: The Role of Network Type. The Journals of Gerontology, Series B: Psychological Sciences and Social Sciences, 72(4), 706-715. doi: 10.1093/geronb/gbw075.

Suanet, B., \& Huxhold, O. (2018). Cohort Difference in Age-Related Trajectories in Network Size in Old Age: Are Networks Expanding ? The Journals of Gerontology, Series B: Psychological Sciences and Social Sciences, gbx166-gbx166. doi: 10.1093/geronb/gbx166.

Will, G. F. (2017). We have an epidemic of loneliness. How can we fix it? The Washington Post, 12. 10. 2017. Wrzus, C., Hanel, M., Wagner, J., \& Neyer, F. J. (2013). Social Network Changes and Life Events Across the Life Span: A Meta-Analysis. Psychological Bulletin, 139(1), 53-80. doi: 10.1037/a0028601. 
Open Access Dieses Kapitel wird unter der Creative Commons Namensnennung 4.0 International Lizenz (http:// creativecommons.org/licenses/by/4.0/deed.de) veröffentlicht, welche die Nutzung, Vervielfältigung, Bearbeitung, Verbreitung und Wiedergabe in jeglichem Medium und Format erlaubt, sofern Sie den/die ursprünglichen Autor(en) und die Quelle ordnungsgemäß nennen, einen Link zur Creative Commons Lizenz beifügen und angeben, ob Änderungen vorgenommen wurden.

Die in diesem Kapitel enthaltenen Bilder und sonstiges Drittmaterial unterliegen ebenfalls der genannten Creative Commons Lizenz, sofern sich aus der Abbildungslegende nichts anderes ergibt. Sofern das betreffende Material nicht unter der genannten Creative Commons Lizenz steht und die betreffende Handlung nicht nach gesetzlichen Vorschriften erlaubt ist, ist für die oben aufgeführten Weiterverwendungen des Materials die Einwilligung des jeweiligen Rechteinhabers einzuholen. 